\title{
Towards Perception-Inspired Numerical Measures of Compression Error in Digital Holograms of Natural Three-Dimensional Scenes
}

\author{
Taina M. Lehtimäki, Ronan G. Reilly, and Thomas J. Naughton \\ Department of Computer Science, Maynooth University, Maynooth, County Kildare, Ireland
}

\begin{abstract}
We report on a visual perception study to measure differences between numerical error in reconstructions from digital holograms of real-world objects that have undergone lossy compression, and the loss in quality perceived by human observers. ㅇ 2018 The Author(s)
\end{abstract}

OCIS codes: $090.1995,100.2000,330.5020$

\section{Introduction}

There is interest in measuring the quality in reconstructions from digital holograms [1], for example in the design of lossy compression algorithms. The quality is typically evaluated with numerical methods (e.g. mean square error). In this study, we evaluate how each of 17 viewers compared the visual quality [2] of reconstructions from lossy compressed and uncompressed holograms, and quantify the differences between perceived error and numerical error. Visual perception studies have been performed before, for example on holographic video of real-world 3D scenes [4].

\section{Digital hologram compression}

Hologram compression differs to that of conventional images in three important respects: holograms can be complexvalued, are Fresnel domain- rather than image domain-signals, and contain multiplicative speckle noise. We used complex-valued phase-shift holograms of 3D objects $[1,3]$. In this paper, we show the results of employing uniform Fresnel-domain quantization. Normalized numerical rms error was calculated between a reconstruction from the quantized hologram and comparing with an equivalent perspective and distance from the original hologram.

\section{Discussion}

Analysis of the results presented in Fig. 1 show that numerical measures of error are sensitive to speckle noise, and that differences in speckle (before and after lossy compression) can lead to a relatively high numerical error even with low compression levels. However, these differences in speckle are not always perceived by a viewer, who may conclude that both holograms are equally noisy and thus have equal quality. In some cases, highly compressed holograms with high numerical error were perceived as having the same quality as the originals. In other cases, even marginallycompressed holograms were perceived by a significant proportion of observers (more than 50\%) as having lower quality than the original. We conclude that simple rms-type numerical measures do not reflect well the perceptual quality of the compressed hologram. This, we believe, is because lossy compression changes the characteristics of the speckle noise in holograms. We propose that modifications to the standard numerical error measures for digital holograms should be made so that numerical measures would be brought closer to the perceived error.

Acknowledgements This publication has emanated from research conducted with the financial support of Science Foundation Ireland (SFI) under Grant Number 13/CDA/222.

\section{References}

1. Th. Kreis, Handbook of Holographic Interferometry: Optical and Digital Methods, Wiley (2005).

2. M. P. Eckert and A. P. Bradley, "Perceptual quality metrics applied to still image compression," Signal Processing 70, 177-200 (1998).

3. Y. Frauel, T. J. Naughton, O. Matoba, E. Tajahuerce, and B. Javidi, "Three-dimensional imaging and processing using computational holographic imaging," Proc. IEEE 94, 636-653 (2006).

4. M. Paturzo, P. Memmolo, A. Finizio, R. Näsänen, T. J. Naughton, and P. Ferraro, "Synthesis and display of dynamic holographic 3D scenes with real-world objects," Opt. Express 18 8806-8815 (2010). 


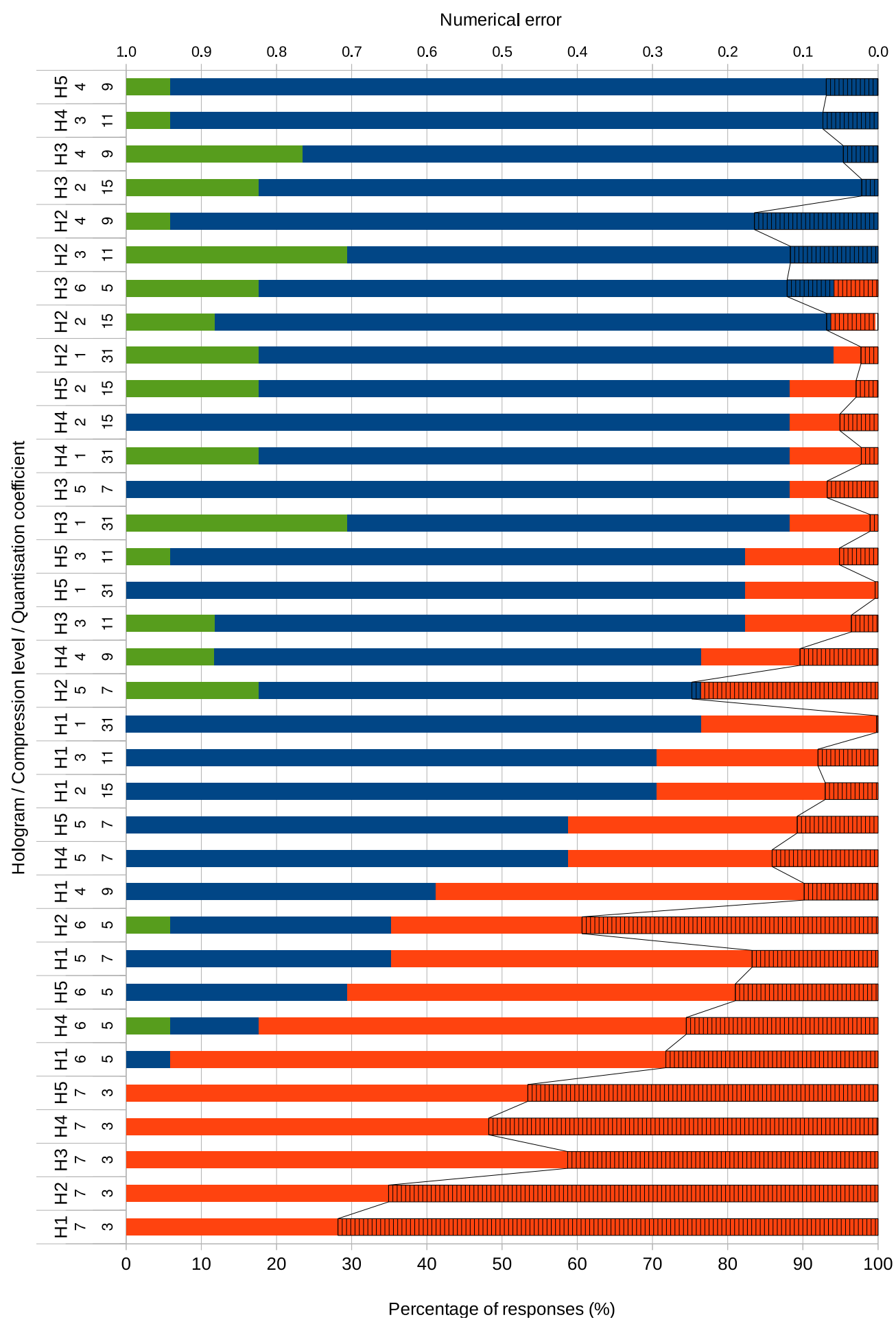

Fig. 1. Differences between numerical error and perceived error. For each hologram (H1 though H5) and each uniform quantization level (1 through 7), the percentage of responses of 17 participants is shown with coloured bars. Green bars indicate that compression had the effect of increasing the perceived quality of the hologram, blue bars indicate that the compression was visually lossless, and red bars indicate that for that particular hologram and compression level, compression caused a decrease in quality. The black lines show numerical rms error in the primary reconstruction plane. The numerical error is inconsistent with the perceived error by our participants. 